\title{
BMJ Open A Qualitative study assessing organisational readiness to implement caregiver support programmes in Ontario, Canada
}

\author{
Victrine Tseung, ${ }^{1}$ Susan Jaglal, ${ }^{2,3}$ Nancy Margaret Salbach (D) ,2,3 \\ Jill I Cameron (1) ${ }^{3,4}$
}

To cite: Tseung V, Jaglal S, Salbach NM, et al. A Qualitative study assessing organisational readiness to implement caregiver support programmes in Ontario, Canada. BMJ Open 2020;10:e035559. doi:10.1136/ bmjopen-2019-035559

- Prepublication history and additional material for this paper are available online. To view these files, please visit the journal online (http://dx.doi. org/10.1136/bmjopen-2019035559).

Received 06 November 2019 Revised 04 April 2020 Accepted 14 April 2020

A) Check for updates

(c) Author(s) (or their employer(s)) 2020. Re-use permitted under CC BY-NC. No commercial re-use. See rights and permissions. Published by BMJ.

${ }^{1}$ Rehabilitation Sciences Institute, Faculty of Medicine, University of Toronto, Toronto, Ontario, Canada

${ }^{2}$ Physical Therapy, University of Toronto, Toronto, Ontario,

Canada

${ }^{3}$ Toronto Rehabilitation Institute, Toronto, Ontario, Canada ${ }^{4}$ Department of Occupational Science and Occupational Therapy, Faculty of Medicine, University of Toronto, Toronto, Ontario, Canada

Correspondence to

Dr Jill I Cameron;

jill.cameron@utoronto.ca

\section{ABSTRACT}

Objective To qualitatively explore factors affecting implementation of caregiver support programmes in healthcare institutions in a regional stroke system. Design A qualitative descriptive study with the Ontario Stroke System (OSS) was conducted. Data were collected through focus groups and in-depth interviews. Transcripts were coded and analysed using inductive thematic analysis.

Setting Regional Stroke System, Ontario, Canada. Participants OSS stakeholders including medical directors, executives, programme directors, education coordinators, rehabilitation and community and long-term care specialists, primary care leaders and healthcare professionals.

Intervention Not applicable.

Main outcome measures Data collection explored perceptions of the need for caregiver support programmes and factors that may affect their implementation.

Results Four focus groups $(n=43)$ and 29 interviews were completed. Analyses identified themes related to (1) evidence that a caregiver programme will improve health and health system outcomes, (2) personnel requirements, (3) barriers associated with current billing and referral processes and (4) integration with current practice and existing workflow processes.

Conclusions Implementation strategies to adopt caregiver programmes into clinical practice should incorporate evidence and consider personnel and existing workflow processes.

\section{INTRODUCTION}

Family caregivers play a central role in the recovery, rehabilitation and community reintegration of individuals who experience a stroke. Numerous studies have documented the negative impact providing care can have on caregiver health and well-being. ${ }^{12}$ Previous literature suggests that $12 \%-25 \%$ of caregivers experience depression ${ }^{34}$ and caregiver burden is positively associated with depression symptoms. ${ }^{5}$ Taking on the caregiver role can also impact other aspects of a caregiver's life, including leisure, relationships and work.

\section{Strengths and limitations of this study}

- Our study used in-depth qualitative methods to explore organisational considerations for implementing caregiver programmes in a regional stroke system.

- Our study included 72 participants reflecting key stakeholder groups responsible for supporting caregivers and implementing caregiver programmes within institutions.

- We did not systematically obtain data related to years of experience in specific roles.

- Additional stakeholder groups (eg, hospital managers) could have provided additional perspectives on caregiver programme implementation.

- More in-depth probing of the specific challenges experienced in rural areas would have expanded our findings.

Relatives who assumed the caregiver role have increased responsibilities, decreased leisure activities and negative changes in their relationships, and the impact on their lives can still be present after a year poststroke. ${ }^{6}$ A study conducted in the USA found that the indirect costs associated with caregiving (eg, travelling time, out-of-pocket expenses) amounted to US $\$ 5669$ per caregiver per year, and costs of total lost productivity amounted to more than US $\$ 10000$ per year per employed caregiver. ${ }^{7}$ Taken together, the caregiver role affects many aspects of a caregiver's life and education, and support has the potential to enhance their abilities to take on and sustain this important role.

Previous literature recommends the use of multicomponent interventions ${ }^{8}$ that cross the care continuum ${ }^{910}$ to meet caregivers' needs for support. Multicomponent interventions include at least two of the following: information provision (eg, community resources), emotional support and counselling (eg, 
support groups, professional support) and skills training (physical care, problem-solving) ${ }^{8}$ For example, the 'Timing It Right' Family Stroke Family Support programme is a multicomponent intervention that is delivered by a healthcare professional (HCP) (stroke support person) who meets the caregiver in-person in acute care and then follows up with six monthly telephone meetings to discuss caregiver's support needs and potential strategies to meet these needs. ${ }^{9}{ }^{10}$ However, caregivers are not benefiting from these programmes, as they are not being incorporated into standard clinical practice. ${ }^{11} 12$ Research from the United Kingdom identified the programme and system-level factors that limited the implementation of a stroke caregiver programme into clinical practice. ${ }^{13} 14$ Participants reported lack of time and individuals responsible for ensuring implementation as limiting factors.

Caregiver programme implementation has been studied in the context of dementia caregiving. Although there are more than 200 efficacious dementia caregiver programmes, only 6 have been integrated into practice. ${ }^{15}$ The need to identify contextual factors that may influence implementation has emerged as an emerging research field and an important step towards effective integration into practice. ${ }^{15}$ Previous studies undertook process evaluations during caregiver programme implementation. ${ }^{131416}$ However, the current study aimed to identify factors that may influence implementation in real-world settings prior to developing or testing an implementation strategy. ${ }^{17}$ Our research to date with the Ontario Stroke System $(\mathrm{OSS})^{18}{ }^{19}$ has indicated a need to understand implementation of caregiver programmes within institutions. Therefore, the objective of this qualitative descriptive study was to examine programme-level factors (ie, within institutions) that influence local implementation of caregiver programmes in the context of a regional stroke system. For the purposes of this study, a caregiver education and support programme was defined as a structured, time-limited intervention with a basic protocol and programme materials. A programme consists of one or more of the following: education, training and support.

\section{METHODS \\ Design}

We conducted a qualitative descriptive study $^{20}$ and used the Standards for Reporting Qualitative Research reporting guidelines. ${ }^{21}$ A qualitative approach is used when the purpose is to understand a specific phenomenon for which there has been limited research and the important variables to explore are unclear. ${ }^{22}$ There are no previous studies that have examined the implementation of caregiver education and support in standard clinical practice within the Canadian context. Specifically, a qualitative descriptive study design is particularly suited when producing a comprehensive description of participant perceptions of a specific phenomenon because data analysis and interpretation stay close to the data and participant perceptions are considered to be accurate and truthful reflections of the reality of the phenomenon (ie, caregiver programme implementation). ${ }^{20}$ Participants were recruited from the OSSregional system of stroke care in the province of Ontario. The OSS is publicly funded, includes 11 geographical regions and provides specialised stroke care across the continuum. Each region includes a regional stroke centre or enhanced district stroke centre, community hospitals, rehabilitation hospitals, community-based providers and prehospital providers. ${ }^{23}$ Some of the regions are large encompassing rural areas and some are small and urban. There are 9 regional stroke centres, 2 enhanced district stroke centres, 16 district stroke centres and 24 secondary prevention clinics. ${ }^{23}$ All participants provided written consent. This study was situated in the Knowledge-toAction Framework's ${ }^{24}$ barrier assessment step to identify facilitators and barriers in the local context. The Consolidated Framework for Implementation Research (CFIR) ${ }^{25}$ informed data collection.

\section{Patient and public involvement}

Patients and the public were not involved in the design of this research. Participants, as described below, were involved in this research through in-depth interviews and focus groups (FG) where they contributed their thoughts about caregivers and programme implementation. Findings have been presented at local and national stroke conferences which are commonly attended by many participants and they will receive copies of publications.

\section{Participants}

Participants were recruited from the 11 OSS regions including: programme directors, rehabilitation specialists, community and long-term care specialists, education coordinators, medical directors (MD) and HCP providing stroke care in acute, rehabilitation and community settings and from the broader healthcare systems including regional health executives (RE) and primary care leaders (PL). Regional rehabilitation specialists, professional education coordinators, community and long-term care specialists and regional/district programme directors were invited by the corresponding author via email to participate in a FG as part of regularly scheduled group meetings. Regional medical directors, health executives and PL were invited by the corresponding author via email to participate in an interview. OSS education coordinators assisted with recruitment of HCP by emailing the study invitation to attendees of their education sessions. The primary author then coordinated and conducted all interviews and FG.

None of the members of the research team had any formal relationships with the participants. The corresponding author has shared their research with members of the OSS through invited presentations and workshops.

\section{Data collection}

Participants were asked to discuss: (1) perceptions of the availability, importance and effectiveness of caregiver 
programmes, (2) current practices related to caregiver education and support and (3) programme-level implementation considerations. The FG and interview questions incorporated the domains of the $\mathrm{CFIR}^{25}$ to explore how individual-level and institution-level factors influence caregiver programme implementation (see online supplementary appendix). For example, individual-level factors such as understanding of caregiver needs, the institution's ability to address caregiver needs and the evidence base for caregiver programmes can all influence programme implementation. ${ }^{25}$ Participants were asked to reflect on all caregiver programmes in general and then more specifically on caregiver programmes that cross care environments using the Timing It Right Stroke Family Support Program ${ }^{910}$ as an example. FG were conducted in person at the local Heart and Stroke Foundation office and interviews were conducted by telephone. The primary author collected all data and is an occupational therapist and doctoral candidate with training in qualitative interviewing. Data collection took place over the course of 4 months and ended when data saturation was reached. Qualitative, semistructured interviews and FG were audio-recorded, transcribed verbatim, reviewed for accuracy and deidentified.

\section{Data analysis}

Qualitative thematic analysis is commonly used to analyse data in qualitative descriptive studies..$^{20}$ Data were analysed using inductive thematic analysis ${ }^{26}$ and facilitated using NVivo. ${ }^{27}$ The first author coded all of the data and all authors reviewed the data and contributed to theme generation. Reflexive notes and field notes were taken throughout data collection and analysis to ensure the dependability and confirmability of the findings. ${ }^{20}$ To maximise the credibility of the findings, all authors reviewed data and contributed to theme generation. ${ }^{28}$

\section{RESULTS}

Four FG ( $\mathrm{n}=43)$ and 29 individual interviews yielded 72 participants (see tables 1 and 2). Four themes related to: (1) need for evidence that a caregiver programme will improve patient and caregiver health and health system outcomes after 'real world' implementation, (2) personnel requirements, (3) barriers associated with current billing and referral processes and (4) integration with current practice and workflow processes. Participant quotations from FG and interviews with HCP, MD, RE and PL are identified by number.

Theme 1: need for evidence that a caregiver programme will improve patient and caregiver health and health system outcomes after 'real-world' implementation

Participants discussed the role of evidence, including programme evaluation, to support funding, implementation and sustainability of caregiver programmes. Participants suggest stakeholders differ in their preferences for process (eg, programme attendance) or outcomes (eg,
Table 1 Professional role of participants: a summary of study participants' professional roles

Number (\%) of participants

Participant group $(\mathrm{n}=72)^{\star}$

Regional/district programme directors (FG) 19 (26)

Community and long-term care specialists 7 (10) (FG)

$\begin{array}{lc}\text { Regional education coordinators (FG) } & 7(10) \\ \text { Regional rehabilitation specialists (FG) } & 11(15) \\ \text { Regional medical directors (INT) } & 2(3) \\ \text { Health region executives (INT) } & 4(6) \\ \text { Health region primary care leads (INT) } & 4(6) \\ \text { Healthcare professionals (INT) } & 19(26)\end{array}$

*One participant had a dual role.

FG, focus group; INT, interview.

health and health system outcomes) data. For example, some prefer numerical data to support implementation (eg, programme attendance) while others are looking for success stories (eg, patient stories). The following quotation describes the roles of pilot testing and programme evaluation to sustain implementation in practice:

But the point I'm trying to get across is that...the organization if they see...there's research to back up the effectiveness of it [caregiver program]...then they'll pilot it... to see if it's working, and then...upper management...they'll look at the number of people who are taking advantage of the programs cause it all comes down to numbers for organizations, right.

Table 2 Healthcare professionals by sector and occupation: a summary of study participants' clinical sector and profession

\begin{tabular}{lc} 
Healthcare professionals by sector and occupation \\
\hline & $\begin{array}{l}\text { Number (\%) of } \\
\text { participants (n=19) }\end{array}$ \\
\hline $\begin{array}{l}\text { Sector } \\
\text { Acute }\end{array}$ & $5(26)$ \\
Rehabilitation & $7(37)$ \\
Community & $6(32)$ \\
Private & $1(5)$ \\
Profession & $5(26)$ \\
Occupational therapist & $4(21)$ \\
Physical therapist & $3(16)$ \\
Speech language pathologist & $3(16)$ \\
Physiatrist/recreational \\
therapist/stroke care \\
navigator
\end{tabular}


And...if it is working right then that pilot program can become...a regular program that the hospital runs and incorporates as a part of their budget. (HCP 19)

\section{Theme 2: personnel requirements}

Many participants agreed that existing team members would not be able to assume this added responsibility, so a new role was needed. In terms of creating a new role, three considerations were discussed: (1) similarity with existing roles, (2) integration into a community-based navigator role and (3) integration into healthcare teams in acute and rehabilitation settings. A few participants highlighted the overlap with existing roles (eg, community care case managers), so this role would need to be clearly defined:

...defining whose role is what and again who's responsible for what, because if you're doing a quarter of the discharge planner's job and you're doing thirty percent of the family doctor's job...there's too much duplication in the hospital and in the health care. (HCP 15)

Some participants spoke about the navigator role in which an individual supports patients and families in the community. Perspectives differed as to the possibility of integrating caregiver support into this role as their availability and function varied by region: '...not every region has a navigator... and those that do they function very differently' (FG 3).

Participants questioned the feasibility of having a position that crosses care environments because current practice does not include contact with patients and their caregivers after they leave the institution. While they indicated the desire for a single point of contact for caregiver support, they spoke about the challenge of determining funding sources and sustainability with current fiscal restraints. The following quotation raises the issue of funding and ownership for this role, particularly in cases where acute and rehabilitation care are separate institutions:

This acute person follows the patient through their rehab stay to community...how does that work when it's [rehab] a separate facility? Like the ownership... that's why the idea of dedicated resources seems to be a good fit to overcome that barrier because if you think about it, if someone's being paid by [hospital A] to handle [hospital A] patients and....and they're off to another...that's what I'm thinking is a barrier for you... (FG 4)

The increasingly large caseload this single person would have to manage was also mentioned as a concern for the feasibility of this approach: '.. just by the nature of the beast it's most likely that you're going to...need a few of these individuals...because their caseload is going to just get bigger and bigger...' (FG 4). Instead, some participants suggested that caregiver support be organised by care environment with healthcare teams picking up the education where the last team finished. The following quotation provides an example: 'I can see it working as...the stroke nurse's role to take it from point A to point B and then...it would be the social worker and rehab's role to be from this section to this section and then it would be the community navigator or the outpatient OT...to be taken to the next stage...' (FG 3).

In addition, some participants felt that it may be more likely to be funded if it was used region wide. However, in geographically larger stroke regions, it would be very challenging for one individual to cover the entire region:

And the individual has to be a regional resource vs organizational. So they can't necessarily belong to a specific hospital but they have to have the capacity to cover a region and be flexible in that... which is a barrier up north. (FG 2)

Participants had different perspectives regarding the role of HCP in providing caregiver support. This individual would need to be knowledgeable about acute care, rehabilitation and community care and to stay up-to-date with community resources. He or she would have counselling skills and a good understanding of each of the disciplines on the healthcare team. Some participants provided examples of specific HCP they perceived to be most appropriate for this role, including occupational therapists, social workers, nurses and other allied health professionals.

Participants questioned the use of trained volunteers or non-health care professionals. Some of the RE discussed the limited availability and high costs of HCP as the main reasons to consider volunteers. The following quotation highlights this point: 'So it may be a costly model if the person is a health care professional. There may be other models where you use peers, somebody who's had a similar experience or volunteers who have been trained' (RE 4). However, there might be instances when the issues facing the families are predominantly medical in nature, and this personnel may not be able to address such issues:

...the challenge would be and we've seen it with community navigators when it's an orphan patient and we have a community navigator following them but all their issues are medical...that community navigator is not equipped to provide advice or to plan any intervention other than to say go to a walk in clinic or et cetera. And that then it becomes a challenge for both parties. (FG 1)

\section{Theme 3: barriers associated with current billing and referral processes}

Participants agreed that the existing infrastructure should incorporate caregiver programmes. However, participants identified several challenges, HCP and rehabilitation specialists suggested that organisational referral criteria and government-funded health insurance preclude caregivers 
from being formal units of care. One participant explained that there are community-based activities that would be appropriate for the patient-caregiver dyad, but because the organisation's mandate is to provide services to individuals with disabilities and there is no billing structure for services provided to caregivers, caregivers are not included. The following quotation demonstrates how the current billing structure and referral criteria hinder the provision of caregiver support within healthcare organisations:

Let's say we register the caregiver as a patient, then they need a chart. Then they need space in health records. Like then we have to track them that way...if the caregiver now becomes the patient then you need to refer them to mental health if that's the issue. Do you know what I mean... So whether or not our institution would adopt that would be really interesting to see. I doubt it...Cause then a cost comes in. (HCP 10)

\section{Theme 4: integration with current practice and existing workflow processes}

Participants discussed four facilitators to the integration of caregiver support into current practice and existing workflow processes. Potential challenges were also discussed.

First, participants suggested that the caregiver support role should become part of routine practice. This approach may overcome limited therapist time to provide support in both inpatient and community care.

Second, participants were concerned with incorporating caregiver support into patient charts. A few participants indicated that there are no places in patient charts to document the specific education provided. If education became required practice, the tendency might be to provide the support for the sake of checking off the box as opposed to paying attention to the content, timing or the readiness of the caregiver to receive this information. The following quotation underscores the challenges with incorporating caregiver support into existing documentation, particularly when these programmes cross care environments:

...so if there is a way to [tick off] have you done your section of [caregiver program]

...you would almost have to be embedded in documentation somehow too so that it is not missed and that would be hard because... once that patient is gone, so is their chart...you are not even supposed to go into the chart after... (FG 3)

Third, participants emphasised communication between the individual providing caregiver support and the rest of the care team to ensure consistency and continuity in messaging to caregivers. This was noted as a challenge in community care because team members do not necessarily communicate with one another or know who else is working with the patient.

Finally, participants highlighted the importance of having a formalised and structured approach to guide implementation. This would require ready-made materials that can be adapted to local contexts, developing a clear referral and monitoring system, setting target user criteria and establishing a mechanism for reaccessing caregivers if they initially decline the programme. Participants suggested that some healthcare professions would need education and tools to assess caregivers' needs and provide appropriate supports. Training may also enhance soft skills as many HCP may be more comfortable with the more technical aspects of their clinical work.

\section{DISCUSSION}

The objective of this study was to identify factors that influence implementation of caregiver programmes within institutions. The four themes suggest that the availability of evidence to support programme outcomes, personnel requirements and a supportive structure including billing, workflow and resources are important for caregiver programme implementation.

As we aim to support transitions across the care continuum, ${ }^{9} 102930$ our findings suggest one potential approach to implementing a programme that crosses care environments. This would involve offering a programme across care environments with healthcare teams picking up the education where the last team finished. Caregivers, however, prefer having one individual provide support across the care continuum. ${ }^{31}$ One possible solution to address these differences in perspectives comes from the ageing literature. Stroke care could adopt an integrated service delivery model such as one proposed for the frail elderly. The Program of Research to Integrate the Services for the Maintenance of Autonomy (PRISMA) model uses case managers to coordinate and monitor the needs of frail elderly across care environments and has demonstrated positive impacts on patient and caregiver health and healthcare utilisation. ${ }^{32}{ }^{33}$ The applicability of this service delivery model to stroke care warrants further consideration.

Participant responses indicated that various HCP would be suitable to provide support to caregivers. One example is occupational therapists where the previous literature suggests that occupational therapists can serve an important role in addressing the needs of caregivers. ${ }^{34}$ Occupational therapists are a core member of multidisciplinary stroke teams and they contribute to caregiver training (eg, teaching activities of daily living (ADLs). ${ }^{35} 36$ Beyond training in ADLs, occupational therapists' training in mental health and meaningful occupations prepare them for addressing the psychosocial effects of caregiving. ${ }^{35}$ There is a need to address caregivers' needs such that they are able to maintain both their own health and engagement in valued activities and provide care for their loved ones. ${ }^{6}$ This previous research ${ }^{6}$ and our study findings highlight occupational therapists as a potential source of support for caregivers.

Current billing structures and referral criteria preclude the provision of services to caregivers in standard clinical practice. This suggests a need to expand organisational structures for billing, referral and documentation to include caregiver support. As demonstrated in the PRISMA model, 
information systems (eg, computerised client chart that is available across organisations) can be developed to overcome organisational structures. ${ }^{32}$ The integration of caregivers into information systems can facilitate programme implementation. ${ }^{14}$

\section{Strengths and limitations}

Our study used in-depth qualitative methods to explore organisational considerations for implementing caregiver programmes in a regional stroke system. Our study included 72 participants reflecting key stakeholder groups responsible for supporting caregivers and implementing caregiver programmes within institutions. We did not systematically obtain data related to years of experience in specific roles. Additional stakeholder groups (eg, hospital managers) could have provided additional perspectives on caregiver programme implementation. More in-depth probing of the specific challenges experienced in rural areas would have expanded our findings.

In summary, our study was one of the first to examine implementation of caregiver programmes within a regional stroke system. It adds to the existing literature by delineating implementation considerations and highlighting the potential role for occupational therapists and other healthcare professionals as providers of caregiver support.

\section{Twitter Nancy Margaret Salbach @nancysalbach}

Contributors VT contributed to the project conceptualisation and design, data acquisition and qualitative interviewing, coding and data analysis, theme generation, drafting and revising the manuscript. SJ and NMS contributed to project conceptualisation and design, qualitative data analysis, theme generation and revisions to the final manuscript. JIC contributed to the project conceptualisation and design, qualitative data analysis, theme generation, drafting and revising the manuscript.

Funding This work was supported by a Ministry of Research and Innovation Early Researcher Award (JIC), Queen Elizabeth II Graduate Student Award in Science and Technology (VT) and a Knowledge Translation Canada Student Fellowship (VT).

\section{Competing interests None declared.}

Patient and public involvement Patients and/or the public were not involved in the design, or conduct, or reporting, or dissemination plans of this research.

Patient consent for publication Not required.

Ethics approval Institutional ethics boards, including the University of Toronto Research Ethics Board, approved the study.

Provenance and peer review Not commissioned; externally peer reviewed.

Data availability statement Data are available upon reasonable request. Data may be accessed by contacting the corresponding author.

Open access This is an open access article distributed in accordance with the Creative Commons Attribution Non Commercial (CC BY-NC 4.0) license, which permits others to distribute, remix, adapt, build upon this work non-commercially, and license their derivative works on different terms, provided the original work is properly cited, appropriate credit is given, any changes made indicated, and the use is non-commercial. See: http://creativecommons.org/licenses/by-nc/4.0/.

\section{ORCID iDs}

Nancy Margaret Salbach http://orcid.org/0000-0002-6178-0691

Jill I Cameron http://orcid.org/0000-0003-4161-1572

\section{REFERENCES}

1 Rigby H, Gubitz G, Phillips S. A systematic review of caregiver burden following stroke. Int J Stroke 2009;4:285-92.
2 Gaugler JE. The longitudinal ramifications of stroke caregiving: a systematic review. Rehabil Psychol 2010;55:108-25.

3 Roth DL, Haley WE, Sheehan OC, et al. Depressive symptoms after ischemic stroke: population-based comparisons of patients and caregivers with matched controls. Stroke 2020;51:54-60.

4 Rohde D, Gaynor E, Large M, et al. Stroke survivor cognitive decline and psychological wellbeing of family caregivers five years poststroke: a cross-sectional analysis. Top Stroke Rehabil 2019;26:180-6.

5 Del-Pino-Casado R, Rodríguez Cardosa M, López-Martínez C, et al. The association between subjective caregiver burden and depressive symptoms in carers of older relatives: a systematic review and metaanalysis. PLoS One 2019;14:e0217648.

6 Pellerin C, Rochette A, Racine E. Social participation of relatives post-stroke: the role of rehabilitation and related ethical issues. Disabil Rehabil 2011;33:1055-64.

7 Ganapathy V, Graham GD, DiBonaventura MD, et al. Caregiver burden, productivity loss, and indirect costs associated with caring for patients with poststroke spasticity. Clin Interv Aging 2015;10:1793-802.

8 White CL, Cantu AG, Trevino MM. Interventions for caregivers of stroke survivors: an update of the evidence. Clin Nurs Stud 2015;3:cns.v3n3p87.

9 Cameron JI, Naglie G, Gignac MAM, et al. Randomized clinical trial of the timing it right stroke family support program: research protocol. BMC Health Serv Res 2014;14:18.

10 Cameron JI, Naglie G, Green TL, et al. A feasibility and pilot randomized controlled trial of the "Timing it Right Stroke Family Support Program". Clin Rehabil 2015;29:1129-40.

11 Rochette A, Korner-Bitensky N, Desrosiers J. Actual vs best practice for families post-stroke according to three rehabilitation disciplines. $J$ Rehabil Med 2007:39:513-9.

12 Rochette A, Racine E, Lefebvre H, et al. Actual and ideal services in acute care and rehabilitation for relatives post-stroke from three perspectives: relatives, stroke clients and health professionals. $J$ Rehabil Med 2014;46:16-22.

13 Clarke DJ, Hawkins R, Sadler E, et al. Introducing structured caregiver training in stroke care: findings from the TRACS process evaluation study. BMJ Open 2014;4:e004473.

14 Clarke DJ, Godfrey M, Hawkins R, et al. Implementing a training intervention to support caregivers after stroke: a process evaluation examining the initiation and embedding of programme change. Implement Sci 2013;8:96.

15 Gitlin LN, Marx K, Stanley IH, et al. Translating evidence-based dementia caregiving interventions into practice: State-of-the-Science and next steps. Gerontologist 2015;55:210-26.

16 Paone D. Using RE-AIM to evaluate implementation of an evidencebased program: a case example from Minnesota. J Gerontol Soc Work 2014;57:602-25.

17 Corporation CfDCaPtK-C. Assuring Healthy Caregivers, a Public Health Approach to Translating Research into Practice:. In: The REAlM framework. Neenah, WI, 2008.

18 Tseung V, Jaglal SB, Salbach NM, et al. Implementing caregiver support programs in a regional stroke system. Stroke 2019;50:STROKEAHA119026660:3585-91.

19 Tseung V, Jaglal SB, Salbach NM, et al. Key informants' perspectives on implementing caregiver programs in an organized system of stroke care. Disabil Rehabil 2019:1-8.

20 Sandelowski M. What's in a name? qualitative description revisited. Res Nurs Health 2010;33:77-84

21 O'Brien BC, Harris IB, Beckman TJ, et al. Standards for reporting qualitative research: a synthesis of recommendations. Acad Med 2014;89:1245-51.

22 Creswell JW. Research Design. In: Qualitative, quantitative and mixed method approaches. 2nd edn. Thousand Oaks, CA: Sage Publications Inc, 2003.

23 Lewis M, Trypuc J, Lindsay P, et al. Has Ontario's stroke system really made a difference? Healthc Q 2006;9:50-9.

24 Graham ID, Logan J, Harrison MB, et al. Lost in knowledge translation: time for a MAP? J Contin Educ Health Prof 2006;26:13-24.

25 Damschroder LJ, Aron DC, Keith RE, et al. Fostering implementation of health services research findings into practice: a consolidated framework for advancing implementation science. Implement Sci 2009;4:50.

26 Braun V, Clarke V. Using thematic analysis in psychology. Qual Res Psychol 2006;3:77-101.

27 NVivo. NVivo qualitative data analysis software. 10.0 edn. QSR International Pty Ltd, 2012.

28 McReynolds CJ, Koch LC, Rumrill PD. Speaking of research. qualitative research strategies in rehabilitation. Work:A Journal of Prevention, Assessment \& Rehabilitation 2001;16:57-65. 
29 Cameron JI, Tsoi C, Marsella A. Optimizing stroke systems of care by enhancing transitions across care environments. Stroke 2008;39:2637-43.

30 Cameron Jl, O'Connell C, Foley N, et al. Canadian stroke best practice recommendations: managing transitions of care following stroke, guidelines update 2016. Int J Stroke 2016;11:807-22.

31 Cameron JI, Naglie G, Silver FL, et al. Stroke family caregivers' support needs change across the care continuum: a qualitative study using the timing it right framework. Disabil Rehabil 2013;35:315-24.

32 Hébert R, Durand PJ, Dubuc N, et al. PRISMA: a new model of integrated service delivery for the frail older people in Canada. Int $J$ Integr Care 2003;3:1-8.
33 Hébert R, Raîche M, Dubois M-F, et al. Impact of PRISMA, a coordination-type integrated service delivery system for frail older people in Quebec (Canada): a quasi-experimental study. J Gerontol B Psychol Sci Soc Sci 2010;65B:107-18.

34 Coutinho F, Hersch G, Davidson H. The impact of informal caregiving on occupational therapy: practice review and analysis. Phys Occup Ther Geriatr 2006;25:47-61.

35 Moghimi C. Issues in caregiving: the role of occupational therapy in caregiver training. Topics in Geriatric Rehabilitation 2007;23.

36 Lawson S, Rowe A, Meredith YY. Survey of stroke caregiver training provided by ot, Pt, and Slp across practice settings. Phys Occup Ther Geriatr 2015;33:320-35. 